\title{
Systemic chemotherapy in combination with pericardial window has better outcomes in malignant pericardial effusions
}

\author{
Sezai Çelik, MD, ${ }^{\mathrm{a}}$ Chiara Lestuzzi, MD, ${ }^{\mathrm{b}}$ Eugenio Cervesato, $\mathrm{PhD},{ }^{\mathrm{c}}$ Didier Dequanter, $\mathrm{MD},{ }^{\mathrm{d}}$ \\ Patrizia Piotti, MD, ${ }^{\mathrm{e}}$ Marzia De Biasio, $\mathrm{MD},{ }^{\mathrm{f}}$ and Massimo Imazio, $\mathrm{MD}^{\mathrm{g}}$
}

Objective: Our aim was to compare systemic chemotherapy $(\mathrm{CT})$ with drainage and with pericardial window in
the treatment of neoplastic pericarditis in patients with various malignancies included in the International
Neoplastic Pericarditis Treatment study.

\begin{abstract}
Methods: Patients treated with systemic CT alone (Group A), CT plus drainage (Group B), or CT plus pericardial window (Group C) were included. Treatment response was defined as complete response (ie, no more pericardial effusion or masses), partial response (ie, reduced total score, without requiring further treatments), stable disease (ie, unchanged total score), or progressive disease (ie, increased total score). Patients with partial or complete response were considered responders.
\end{abstract}

Results: This preliminary report included 175 patients (56.6\% male) with a mean age of $54.21 \pm 14.26$ years. Gender distribution, age, and follow-up duration was similar for all groups $(P>.05)$. Prevalent cancer types were lung cancer $(50.9 \%)$, breast cancer $(14.9 \%)$, and lymphoma/leukemia $(14.9 \%)$. Overall, $22.3 \%, 42.9 \%$, and $34.9 \%$ of patients were in treatment group A, B, and C, respectively. There were 132 responders $(75.3 \%)$. The rate of responders significantly differed between groups $(P<.001)$; it was significantly higher in Group B than in Group A $(P<.05)$ and in Group C than in Group B $(P=.006)$. The significant factors affecting response were therapy $(P=.002)$ and extent of effusion $(P=.037)$. Kaplan-Meier analysis showed that patients in Group $\mathrm{C}$ had a significantly better survival rate than patients in the other groups $(P=.001)$.

Conclusions: Systemic CT plus pericardial window is a more effective treatment option compared with systemic CT alone and systemic CT plus drainage in patients with malignant effusions. (J Thorac Cardiovasc Surg 2014;148:2288-93)

See related commentary on pages $2294-5$.

Pericardial effusion is a common problem causing morbidity and mortality in patients with malignancy. The prevalence of cardiac involvement in malignancies is between $5 \%$ and $20 \%$ in autopsy series and clinical studies. $^{1-4}$ The most common malignancies with pericardial effusions are lung carcinoma, lymphoma, leukemia, and breast cancer. ${ }^{2,5,6}$

\footnotetext{
From the Department of Thoracic Surgery, ${ }^{\text {a }}$ Siyami Ersek Thoracic and Cardiovascular Surgery Training and Research Hospital, İstanbul, Turkey; Cardiology Unit, ${ }^{\text {b }}$ Department of Oncology Centro di Riferimento Oncologica National Cancer Institute, Aviano, Italy; Department of Cardiology, ${ }^{\mathrm{c}} \mathrm{ARC}, \mathrm{S}$ Maria Degli Angeli Hospital, Pordenone, Italy; Department of Surgery, ${ }^{\mathrm{d}}$ Centre Hospitalier Universitaire Charleroi, Montigny le Tielleuil, Belgium; Department of Cardiology, Istituto Nazionale Tumori, National Cancer Institute, Milan, Italy; Department of Cardiology, ${ }^{\mathrm{f}}$ AOU S Maria Della Misericordia, Udine, Italy; and Department of Cardiology, ${ }^{\mathrm{g}}$ Maria Vittoria Hospital, Turin, Italy.

Disclosures: Chiara Lestuzzi reports lecture fees from Novartis. All other authors have nothing to disclose with regard to commercial support.

Received for publication Nov 25, 2013; revisions received March 20, 2014; accepted for publication April 11, 2014; available ahead of print May 15, 2014.

Address for reprints: Sezai Çelik, MD, Kiptaş Yeşilvadi Evleri A3 Blok D 36, 34000 Ümraniye, İstanbul, Turkey (E-mail: siyamie@gmail.com).

$0022-5223 / \$ 36.00$

Copyright (c) 2014 by The American Association for Thoracic Surgery

http://dx.doi.org/10.1016/j.jtcvs.2014.04.031
}

There are several reasons causing pericardial effusions in malignancies. About $30 \%$ of cases are due to radiation therapy; mediastinal lymph node engorgement; systemic tumor treatment; and viral, bacterial, and autoimmune causes. In $70 \%$ of cases the effusion is due to neoplastic invasion of the pericardium, and systemic chemotherapy (CT) as the only treatment, often fails. The presence of a large effusion may require drainage of the fluid to avoid cardiac tamponade, but the effusion tends to relapse if additional treatments are not given. Drainage may be obtained through pericardiocentesis performed by inserting a catheter with the Seldinger technique, or creating a pleuropericardial window. Pericardial window creation is the most common surgical treatment used for neoplastic pericardial effusions in association to systemic CT, but there are a limited number of studies comparing its efficacy to simple pericardiocentesis. The international multicenter retrospective study called International Neoplastic Pericarditis Treatment (INPUT) study was planned to be conducted to evaluate the outcome of a large number of patients with neoplastic pericarditis treated according to the usual care in each institution (systemic CT, local CT, pericardial drainage, and pericardial window). In a preliminary report, the efficacy of 4 treatments alone or in combination (pericardial drainage, sclerosing therapy, or local or systemic CT) in a subgroup of lung cancer patients was analyzed. ${ }^{7}$ In this 

Abbreviations and Acronyms
$\mathrm{CT}=$ chemotherapy
INPUT $=$ International Neoplastic Pericarditis
Treatment study
VATS $=$ video-assisted thoracoscopic surgery

report, only lung cancer patients were included, and none of the patients had been treated by pericardial window, thus leaving the open question if pericardial window is more effective than drainage in preventing recurrences.

The aim our study was to compare the outcome of neoplastic pericarditis treated either with systemic $\mathrm{CT}$ alone (without drainage), with drainage, and with pericardial window formation in patients with various malignancies, among those included in the INPUT study.

\section{MATERIALS AND METHODS}

According to the INPUT study, the neoplastic nature of the effusion has to be confirmed by cytology, histopathology, or elevated tumor markers in patients undergoing drainage. Patients with small pericardial effusion did not undergo pericardial drainage; in those patients, the diagnosis of neoplastic effusion was confirmed by the presence of intrapericardial neoplastic masses (detected by computed tomography or positron emission tomography) or by cytology if the effusion worsened and was finally drained. Patients who died within 15 days (for causes not related to the pericardial procedures) or could not be followed-up were excluded. The ongoing INPUT study is currently including 11 centers from 5 countries. For our study, among the total group of 326 patients we considered the subgroup comprising 175 patients treated by systemic CT alone, without drainage (Group A); CT plus drainage (Group B); or CT plus pericardial window (Group C). We excluded 33 patients who did not receive any CT, and 118 patients who were treated by local CT alone or in combination with systemic CT. This subgroup included the patients observed in 8 centers in 3 countries, namely, Belgium, Turkey, and Italy. All patients received systemic $\mathrm{CT}$ according to their primary tumor and to the state of art at the time of their disease. The choice between catheter drainage and pericardial window depended on the routine approach in different hospitals.

Each patient was treated according to the clinical knowledge at the time of the treatment, to the attitude of cardiologists, oncologists, and surgeons of each hospital. Thus, neither ethic committee approval (no changes from routine local practice), nor informed consent was required (excluding the usual consent for invasive procedures).

The names of patients were obtained from hospital records, and follow-up data were obtained by follow-up appointments and from hospital records. The follow-up was based on imaging techniques (ie, echocardiography, computed tomography scan, magnetic resonance imaging, and positron emission tomography) or autopsy data. Telephone follow-up was used to record the date of death only. The database includes patients seen at Centro di Riferimento Oncologica National Cancer Institute, Aviano, Italy, between 1985 and 2012, and between 1999 and 2012 in the other participating centers.

Pericardiocentesis was performed using the Seldinger technique and inserting a pigtail catheter in the pericardial space. After drainage, the catheter was left in place until no significant amount of fluid was drained.

For the creation of a pericardial window, left anterior minithoracotomy $(4-5 \mathrm{~cm})$ technique was used under general anesthesia. Patients were placed in the left lateral decubitis position. Each patient's chest was opened between the fourth and fifth intercostal spaces. A $4 \times 4 \mathrm{~cm}$ or $5 \times 5 \mathrm{~cm}$ portion of the left pericardium was excised. Fluid and pericardium sample was sent for histologic examination. A drainage tube was inserted into the pleural space and left in place for the following 3 to 4 days.

A scoring system, including both the amount of effusion and the size of the neoplastic pericardial masses was used to assess the disease at presentation and the outcome of the treatment, as already described. ${ }^{7}$ Extent of effusion was graded (effusion score) such that $0=$ no effusion, $1=<1$ $\mathrm{cm}, 2=1$ to $2 \mathrm{~cm}, 3=2$ to $3 \mathrm{~cm}$, and $4=>3 \mathrm{~cm}$ mean separation on apical view (or at $\mathrm{CT}$ scan). Mass score was graded such that $1=$ a single mass $<2 \times 2 \mathrm{~cm} ; 2=$ multiple masses, or a single mass $>2 \times 2 \mathrm{~cm}$ or superficial infiltration; and $3=$ tumor encasement or transmural infiltration.

The outcome was considered at the last available follow-up or at the time when a new treatment (first or new pericardial drainage or pericardial window after drainage) was chosen because of worsening, relapse, or incomplete response. The response was classified as complete response (no more pericardial effusion or masses; score $=0$ ), partial response (reduction of the total score, without requiring further treatments), stable disease (unchanged total score), or progressive disease (increased total score).

Patients who had partial or complete response were considered responders, whereas those with stable disease or progression were considered nonresponders.

\section{Statistical Analysis}

Data were analyzed using the Statistical Package for the Social Sciences version 13 (IBM-SPSS Inc, Armonk, NY). Descriptive statistics were given as mean \pm standard deviation, median, or number (percent). The $\chi^{2}$ test was used to compare the rate of responders in the groups and for post hoc analysis. Forward stepwise logistic regression analysis (Wald method) was used to determine the variables affecting response. Kaplan-Meier survival analysis was performed to determine survival in the groups.

\section{RESULTS}

Our preliminary report included 175 patients $(56.6 \%$ male) with a mean age of $54.21 \pm 14.26$ years (range, 15-84.9 years), from the cardiology, cardiology/oncology, surgery, and thoracic surgery departments of 8 centers from 3 different countries. Most of the patients were enrolled from the cardiology/oncology department $(34.3 \%)$, followed by surgery $(29.1 \%)$, thoracic surgery $(20 \%)$, and cardiology $(16.6 \%)$.

Gender distribution, mean age, and mean follow-up duration was similar for all groups $(P>.05)$. The types of cancer were lung cancer in 89 patients $(50.9 \%)$, breast cancer in 26 patients $(14.9 \%)$, lymphoma/leukemia in 26 patients $(14.9 \%)$, and other types of cancer in 34 patients $(19.3 \%)$. Although there was a somewhat even distribution regarding cancer types in Group A, most of the patients in Group B and C had lung cancer. The demographic characteristics and outcome of the patients are given in Table 1 . Overall, $22.3 \%$ of patients $(\mathrm{n}=39)$ were in Group A, whereas $42.9 \%(n=75)$ and $34.9 \%(n=61)$ of patients were in Group B and Group C, respectively.

The effusion was $<1 \mathrm{~cm}$ in only $16 \%$ of patients, and more than half of patients $(50.9 \%)$ had a single mass $<2 \times 2 \mathrm{~cm}$ (mass score $=1$ ). The extent of effusion (effusion scores) and patients' mass scores are given in Table 2. An effusion score $\geq 3$ was present in 6 patients in Group A, in 56 patients in Group B, and in 31 patients in Group C. In Groups B and C, pericardial drainage in 
TABLE 1. Patient characteristics and outcome, according to group

\begin{tabular}{|c|c|c|c|c|c|c|c|}
\hline & $\begin{array}{c}\text { Group A } \\
(\mathrm{n}=39)\end{array}$ & $\begin{array}{r}\text { Group B } \\
(n=75) \\
\end{array}$ & $\begin{array}{r}\text { Group C } \\
(\mathrm{n}=51)\end{array}$ & $P($ all $)$ & $\begin{array}{c}P \\
(A \text { vs B })^{*}\end{array}$ & $\begin{array}{c}P \\
(\mathbf{A} \text { vs C })^{*}\end{array}$ & $\begin{array}{c}P \\
(\text { B vs C })^{*}\end{array}$ \\
\hline \multicolumn{8}{|l|}{ Sex } \\
\hline Female & $21(53.8)$ & $30(40.0)$ & $25(41.0)$ & .328 & - & - & - \\
\hline Male & $18(46.2)$ & $45(60.0)$ & $36(59.0)$ & & & & \\
\hline Age, y & $51.09 \pm 17.55$ & $55.03 \pm 14.08$ & $55.2 \pm 11.9$ & .302 & - & - & - \\
\hline Follow-up, d & $302.08 \pm 567.1$ & $301.71 \pm 537.45$ & $238.25 \pm 287.94$ & .934 & - & - & - \\
\hline \multicolumn{8}{|l|}{ Site of primary neoplasm } \\
\hline Lung & 7 (17.9) & $47(62.7)$ & $35(57.4)$ & .001 & $<.001$ & .001 & .748 \\
\hline Breast & $10(25.6)$ & $7(9.3)$ & $9(14.8)$ & & & & \\
\hline Lymphoma/leukemia & $11(28.2)$ & $9(12)$ & $6(9.8)$ & & & & \\
\hline Other & $11(28.2)$ & $12(16)$ & $11(18.0)$ & & & & \\
\hline \multicolumn{8}{|l|}{ Response } \\
\hline Yes & $21(53.8)$ & $55(73.3)$ & $56(91.8)$ & $<.001$ & .036 & $<.001$ & .006 \\
\hline No & $18(46.2)$ & $20(26.7)$ & $5(8.2)$ & & & & \\
\hline \multicolumn{8}{|l|}{ Response type } \\
\hline Complete response & $10(25.6)$ & $22(29.3)$ & $19(31.1)$ & $<.001$ & .003 & $<.001$ & .022 \\
\hline Partial response & $11(28.2)$ & $33(44.0)$ & $37(60.7)$ & & & & \\
\hline Stable disease & $4(10.3)$ & $14(18.7)$ & $5(8.2)$ & & & & \\
\hline Progression & $14(35.9)$ & $6(8.0)$ & $0(0.0)$ & & & & \\
\hline \multicolumn{8}{|c|}{$\begin{array}{l}\text { Overall observation time from diagnosis } \\
\text { to last follow-up or to event, } \mathrm{d}\end{array}$} \\
\hline$>30$ & $36(92.3)$ & $65(86.7)$ & $52(85.2)$ & .563 & - & - & - \\
\hline$>60$ & $31(79.5)$ & $52(69.3)$ & $47(77.0)$ & .415 & - & - & - \\
\hline$>180$ & $16(41.0)$ & $27(36.0)$ & $25(41.0)$ & .798 & - & - & - \\
\hline$>365$ & $5(12.8)$ & $13(17.3)$ & $13(21.3)$ & .552 & - & - & - \\
\hline
\end{tabular}

Data are presented as mean \pm standard deviation or $\mathrm{n}(\%)$. *For the post-hoc analysis, $P<.017$ was considered significant.

patients with an effusion score $<3$ was performed for diagnostic purposes or to prevent cardiac tamponade in loculated effusions.

Overall, 132 patients $(75.3 \%)$ showed response to therapy; 51 of whom showed complete response, whereas 81 patients showed partial response. The number of patients who showed response to therapy was $21(53.8 \%), 55$ $(73.3 \%)$, and $56(91.8 \%)$ in Groups A, B, and C, respectively $(P<.001)$. A post-hoc analysis showed that the rate of responders were significantly higher in Group B than in Group A $(P<.05)$ and in Group $\mathrm{C}$ than in Group B $(P=.006)($ Table 1$)$.

A logistic regression analysis was performed to determine the factors affecting response to therapy. Gender, age, mass,

TABLE 2. Effusion and mass scores of the patients

\begin{tabular}{ll}
\hline & No. of patients $(\mathbf{N}=\mathbf{1 7 5})$ \\
\hline Effusion score & \\
1 & $28(16)$ \\
2 & $54(30.9)$ \\
3 & $53(30.3)$ \\
4 & $40(22.9)$ \\
Mass score & \\
1 & $89(50.9)$ \\
2 & $26(14.9)$ \\
3 & $60(34.3)$ \\
\hline
\end{tabular}

Data are presented as $\mathrm{n}(\%)$. extent of effusion, and therapy was entered and it was found that therapy $(P=.002)$ and extent of effusion $(P=.037)$ were the significant factors affecting response, whereas gender, age, and mass of the tumor were not significant.

Kaplan-Meier survival analysis showed that patients in Group $\mathrm{C}$ had a significantly better survival rate than the other groups $(P=.001)$ (Figure 1$)$.

Pericardiocentesis had no major complications. Transitory lowering of blood pressure was successfully cured by leg elevation or intravenous fluid administration. There was no procedure-related mortality and major morbidity in patients undergoing pericardial window formation. Pericardiocentesis was performed in patients with cardiac tamponade to prevent low cardiac output syndrome just before the operation.

\section{DISCUSSION}

Pericardial effusion requiring therapy is not an unusual condition in malignancies. In our study, in which 175 patients with malignant pericardial effusion were included, a new scoring system using the extent of effusion (measured in centimeters), and tumor mass in the equation, defined by Lestuzzi and colleagues, ${ }^{7}$ was used to assess treatment response. Overall, $74.2 \%$ of patients showed complete response to either systemic CT, systemic CT plus drainage, or systemic CT plus pericardial window formation. 


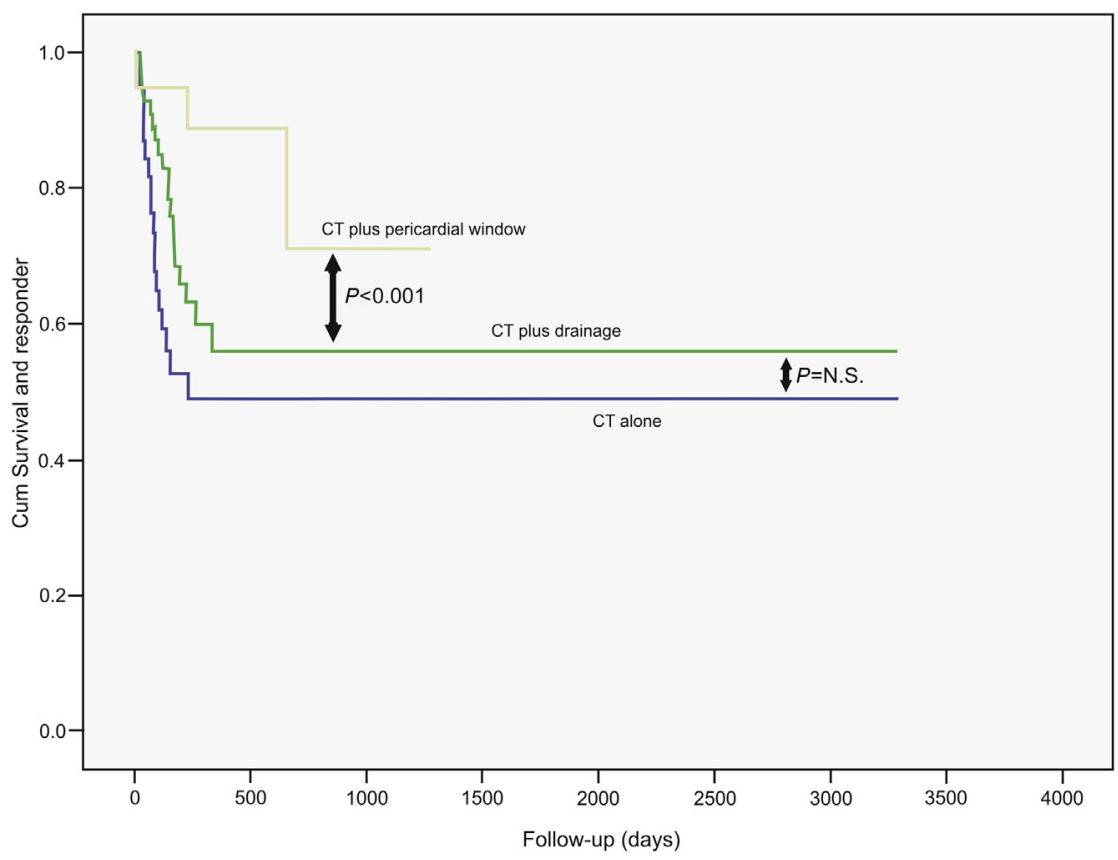

FIGURE 1. Kaplan-Meier survival curve. In 1 case follow-up was truncated to 3290 days; this is the max value plotted. CT, Chemotherapy; N.S., not significant.

According to treatment responses and survival estimations, the best treatment option in this group of patients seems to be pericardial window formation plus systemic CT. The logistic regression analysis showed that extent of effusion and treatment modality used were the significant factors affecting treatment response.

Pericardial involvement is usually secondary to a primary tumor. The most common secondary malignant tumors involving the pericardium are lung cancer, breast cancer, malignant melanoma, lymphoma, and leukemia., ${ }^{2,6}$ As is reported in the literature, more than half of our patients $(50.9 \%)$ were lung cancer patients, followed by breast cancer and lymphoma/leukemia patients at the same rate $(14.9 \%)$.

Various treatment modalities either alone or in combination have been used in the treatment of malignant pericardial effusion in patients with cancer. However, there is still no consensus on the best treatment option, given the wide spectrum of malignancies involving the pericardium, the different causes of pericardial effusion in malignant patients, and the clinical condition of individual patients. The aims of treatment in patients with malignant pericardial effusions include symptom relief, prevention of recurrences, treatment of local disease, and improvement of quality of life. Treatment should prolong survival with minimal procedure-related morbidity and mortality. Immediate relief of symptoms may be obtained with percutaneous drainage or with a surgical approach. For long-term prevention of recurrences, various modalities such as extended drainage, pericardial window (surgical or percutaneous balloon pericardiostomy), local sclerosing therapy, local and/or systemic CT, or radiation therapy, have been used in the literature. ${ }^{1,8,9}$

There is no large-scale study comparing outcomes in patients treated with CT alone. Nevertheless, in a prospective study on 32 patients with non-Hodgkin's lymphoma, 5 of 17 cases with pericardial effusion underwent pericardiocentesis. The authors stated that the presence of pericardial effusion had no adverse effect on survival at 1 year in advanced non-Hodgkin's lymphoma. ${ }^{10}$ Furthermore, Reynolds and colleagues ${ }^{11}$ treated malignant pericardial effusion with a single pericardiocentesis followed by intensive systemic combination CT in 3 patients with metastatic carcinoma of the breast. Available data support the use of systemic CT in previously untreated patients with highly CT-sensitive tumors like lymphoma and leukemia. ${ }^{12}$ According to the response rates obtained in our study, we also think that systemic CT may be effective in treating neoplastic pericarditis due to breast cancer and lymphomas, but has little effect in patients with lung cancer.

Percutaneous pericardiocentesis is usually followed by a definitive medical or surgical procedure designed to prevent further fluid accumulation, to provide an alternative drainage site to prevent cardiac tamponade,,$^{13}$ and to obtain a diagnosis, but have some risks such as cardiac and coronary laceration, pneumothorax, liver trauma, and death. Echocardiographic guidance considerably enhances the safety of this procedure. However, $40 \%$ to $90 \%$ of pericardial effusions will recur within 3 months after pericardiocentesis alone, and extended catheter drainage, which 
reduces the rate of recurrence, is recommended by Mayo Clinic cardiologists as their technique of choice., ${ }^{2,9}$

Studies comparing percutaneous pericardiocentesis with various treatment modalities like pericardial window formation or surgery have contradictory results. Although some researchers report beneficial results and no major procedure-related complications, as do Kabukcu and colleagues, ${ }^{14}$ some report unsuccessful results, requirement of repeat pericardiocentesis, and complications like right ventricular puncture. ${ }^{15-17}$

In patients with symptoms or clinical cardiac tamponade, pericardiocentesis provides symptom relief, but does not improve survival and does have a high recurrence rate.

Echocardiographic-guided pericardiocentesis gave a $74.2 \%$ complete response rate in our study, without any significant procedure-related morbidity with this technique.

The most commonly used surgical approach is pericardiotomy or the creation of a pericardial window connected to a drainage tube or draining into the pleural space (using the subxiphoid approach, a left thoracotomy, video-assisted thoracoscopic surgery [VATS], or a balloon catheter). ${ }^{9}$

The studies using VATS in the treatment of pericardial effusions reported few or no recurrence in their series. ${ }^{18,19}$ However, because VATS requires single-lung ventilation and double-lumen anesthesia, it cannot be performed, especially in patients with hemodynamic instability.

Subxiphoid approach performed under local anesthesia is an easier technique, but it is associated with high recurrence rates. ${ }^{20}$ Therefore, creating a pericardial window by subxiphoid method should not be preferred in patients with a high probability of long-term survival.

Pericardial window via anterior minithoracotomy is associated with lower morbidity, no mortality, and a lower recurrence rate, but recovery is delayed. ${ }^{21,22}$

Cullinane and colleagues ${ }^{23}$ reviewed 62 patients with malignant disease who underwent surgical pericardial window creation for management of pericardial effusions. Windows were created either thoracoscopically $(\mathrm{n}=32)$, by subxiphoid approach $(\mathrm{n}=12)$, or by limited thoracotomy approach $(\mathrm{n}=18)$. Primary tumors included non-small cell lung carcinoma as well as breast, hematologic, and other solid-organ malignancies. Three patients had recurrent effusions $(4.8 \%) ; 2$ in the subxiphoid group and 1 in the thoracoscopy group. The median survival was shorter for patients with non-small cell lung carcinoma (2.6 months) than for patients with breast cancer (11 months) or hematologic malignancy (10 months). The authors concluded that surgical pericardial window is a safe and durable operative procedure that may provide extended survival in certain subgroups of cancer patients. ${ }^{23}$

In 1 of our previous studies, we also investigated the effectiveness of pericardial window formation via minithoracotomy for treating pericardial tamponade in 48 cancer patients. $^{22}$ There was no surgery-related mortality and the 30-day mortality rate was $8.33 \%$ (all during postoperative hospitalization). Morbidity rate was $18.75 \%$. Symptomatic recurrence rate was $2.08 \%$. Cancer type and nature of the pericardial effusion were the major factors determining long-term survival $(P<.001$ and $P<.004$, respectively). As mentioned, the major reason for preferring left minithoracotomy for creating a pericardial window in our clinic is that this group of patients needs prompt and definitive diagnosis and treatment. This technique has some advantages, like not requiring single-lung ventilation; it can be performed in only 25 to 30 minutes; and it provides a larger window, thus allows obtaining a larger tissue sample; and it offers the opportunity for pleural biopsy. The most important disadvantage is the risk of sudden hypotension during induction of general anesthesia, but this risk can be overcome by pericardiocentesis performed immediately before the procedure. For this reason, echo-guided pericardiocentesis was performed in all patients before the pericardial window procedure in our study, and no intraoperative problems were encountered in these patients.

However, if pericardiocentesis is easy and safe (ie, there is a safe approach according to echocardiography, with $>2 \mathrm{~cm}$ free space between parietal and visceral pericardium, without interposed organs or masses, either from the subxifoid or apical approach), and there are cardiologists able to do it, percutanous pericardial drainage may be the preferred approach. In case of difficult/harmful approach (eg, enlarged liver, moderate effusion with signs of tamponade, and effusive-constrictive pericarditis) or relapse after pericardiocentesis and chemotherapy, a surgical approach is suggested.

In a previous work, intrapericardial CT with platinum derivatives was significantly superior to systemic $\mathrm{CT}$ in lung cancer neoplastic pericarditis, ${ }^{7}$ and this success was supposed to be due to the local action of platinum within the lymphatic vessels. ${ }^{7}$ This approach is possible only using an intrapericardial catheter. In a more recent report in a larger group of patients with neoplastic pericarditis, ${ }^{24}$ the advantage of intrapericardial CT was confirmed in lung cancer, but not in all kinds of tumors. Our study is the first to compare pericardial drainage and pericardial window outcomes. It might be considered the basis for possible further prospective randomized studies, which would require a large number of institutions all with cardiac or thoracic surgery facilities; a long enrolment period; and the ethical problem of proposing a more invasive approach against a procedure (percutaneous pericardiocentesis) that is usually well tolerated and excluding the use of intrapericardial chemotherapy, which has been shown to be useful in a subgroup of patients with lung cancer.

\section{Study Limitations}

Our study was an observational multidisciplinary study on patients with various malignancies; patient names and characteristics were obtained from hospital records and 
follow-up data were obtained from follow-up appointments and from hospital records. Therefore, there is neither randomization nor standardization of the procedures for practical and ethical reasons; interventions were determined by individual preferences (patient and physician) and practice patterns.

However, our study design allowed us to make comparisons between systemic CT alone, systemic CT plus drainage, and systemic CT plus pericardial window creation. A prospective randomized study would be very hard to perform for practical reasons because hospitals without cardiac or thoracic surgery facilities should send the patients elsewhere, which might be impossible in an emergency. Furthermore, in hospitals where the surgical approach is routinely used, cardiologists may not have adequate skills to perform a percutaneous pericardiocentesis. Furthermore, to increase the validity of our results, only patients with neoplastic effusion confirmed by cytology, histopathology, or elevated tumor markers were included in the study, and we used an objective scoring system to assess the treatment response.

\section{CONCLUSIONS}

According to the results of our study (including 175 patients with neoplastic pericarditis), systemic CT plus pericardial window creation is a more effective treatment option compared to systemic CT alone and systemic CT plus drainage in patients with malignant effusions.

We believe a significant subgroup of patients may improve with treatment including pericardial drainage plus local and/or systemic CT, mostly patients with lung cancer or with highly chemosensitive tumors. Pericardial window creation (even with the mini-invasive method of balloon pericardiostomy) is an effective and safe second line treatment in relapsing effusion after pericardial drainage, and in cases refractory to other therapeutic approaches. It can be used as first-line treatment in patients with malignant neoplasms less likely to be successfully treated by local or systemic CT, such as mesotheliomas and sarcomas, or whenever the possibility of an intrapericardial therapy is excluded by an oncologist.

\section{References}

1. Buzaid AC, Garewal HS, Greenberg BR. Managing malignant pericardial effusion. West J Med. 1989;150:174-9.

2. Grannis FW Jr, Lai L, Kim JY. Fluid complications. http://www.cancernetwork. com/cancer-management/fluid-complications/article/10165/1802878. Accessed April 21, 2014

3. Maisch B, Ristic A, Pankuweit S. Evaluation and management of pericardial effusion in patients with neoplastic disease. Prog Cardiovasc Dis. 2010;53:157-63.
4. MacGee W. Metastatic and invasive tumours involving the heart in a geriatric population: a necropsy study. Virchows Arch A Pathol Anat Histopathol. 1991; 419:183-9.

5. Abraham KP, Reddy V, Gattuso P. Neoplasms metastatic to the heart: review of 3314 consecutive autopsies. Am J Cardiovasc Pathol. 1990;3:195-8.

6. Lam KY, Dickens P, Chan AC. Tumors of the heart. A 20-year experience with a review of 12,485 consecutive autopsies. Arch Pathol Lab Med. 1993;117: 1027-31.

7. Lestuzzi C, Bearz A, Lafaras C, Gralec R, Cervesato E, Tomkowski W, et al. Neoplastic pericardial disease in lung cancer: impact on outcomes of different treatment strategies. A multicenter study. Lung Cancer. 2011; 72:340-7.

8. Tsang TS, Seward JB, Barnes ME, Bailey KR, Sinak LJ, Urban LH, et al. Outcomes of primary and secondary treatment of pericardial effusion in patients with malignancy. Mayo Clin Proc. 2000;75:248-53.

9. Lestuzzi C. Neoplastic pericardial disease: old and current strategies for diagnosis and management. World J Cardiol. 2010;2:270-9.

10. Acquatella GC, Roura ET, Maury AJ, Stern RO, Acquatella H. High incidence of pericardial effusion in non-Hodgkin's lymphoma: usefulness of echocardiography. Eur J Cancer Clin Oncol. 1982;18:1131-6.

11. Reynolds PM, Byrne MJ. The treatment of malignant pericardial effusion in carcinoma of the breast. Aust N Z J Med. 1977;7:169-71.

12. Lamont E, Hoffman PC. Oncologic emergencies. In: Hall JB, Schmidt GA, Wood LDH, eds. Principles of critical care. 3rd ed. New York: McGraw Hill; 2005 .

13. Maher EA, Shepherd FA, Todd TJ. Pericardial sclerosis as the primary management of malignant pericardial effusion and cardiac tamponade. J Thorac Cardiovasc Surg. 1996;112:637-43.

14. Kabukcu M, Demircioglu F, Yanik E, Basarici I, Ersel F. Pericardial tamponade and large pericardial effusions: causal factors and efficacy of percutaneous catheter drainage in 50 patients. Tex Heart Inst J. 2004;31:398-403.

15. Little AG, Kremser PC, Wade JL, Levett JM, DeMeester TR, Skinner DB. Operation for diagnosis and treatment of pericardial effusions. Surgery. 1984;96: 738-44.

16. Laham RJ, Cohen DJ, Kuntz RE, Baim DS, Lorell BH, Simons M. Pericardial effusion in patients with cancer: outcome with contemporary management strategies. Heart. 1996;75:67-71.

17. Gumrukcuoglu HA, Odabasi D, Akdag S, Ekim H. Management of cardiac tamponade: a comparative study between echo-guided pericardiocentesis and surgery-a report of 100 patients. Cardiol Res Pract. 2011;2011: 197838.

18. O’Brien PK, Kucharczuk JC, Marshall MB, Friedberg JS, Chen Z, Kaiser LR et al. Comparative study of subxiphoid versus video-thoracoscopic pericardial "window" Ann Thorac Surg. 2005;80:2013-9.

19. Uramoto H, Hanagiri T. Video-assisted thoracoscopic pericardiectomy for malig nant pericardial effusion. Anticancer Res. 2010;30:4691-4.

20. Mueller XM, Tevaearai HT, Hurni M, Ruchat P, Fischer AP, Stumpe F, et al. Long-term results of surgical subxiphoid pericardial drainage. Thorac Cardiovasc Surg. 1997;45:65-9.

21. Neragi-Miandoab S, Linden PA, Ducko CT, Bueno R, Richards WG, Sugarbaker DJ, et al. VATS pericardiotomy for patients with known malignancy and pericardial effusion: survival and prognosis of positive cytology and metastatic involvement of the pericardium: a case control study. Int J Surg. 2008;6: 110-4.

22. Celik S, Celik M, Aydemir B, Tanrikulu H, Okay T, Tanrikulu N. Surgical properties and survival of a pericardial window via left mini thoracotomy for benign and malignant pericardial tamponade in cancer patients. World J Surg Oncol. $2012 ; 10: 123$.

23. Cullinane CA, Paz IB, Smith D, Carter N, Grannis FW Jr. Prognostic factors in the surgical management of pericardial effusion in the patient with concurrent malignancy. Chest. 2004;125:1328-34.

24. Lestuzzi C, Cervesato E, Lafaras C, Celik S, Dequanter D, Tomkowski W, et al Which is the best approach for neoplastic pericardial effusion? A retrospective analysis of 264 cases. Eur Heart J. 2013;34(Suppl 1):P4483. 